\title{
A CHARACTERIZATION RELATED TO THE EQUILIBRIUM DISTRIBUTION ASSOCIATED WITH A POLYNOMIAL STRUCTURE
}

\author{
SHAUL K. BAR-LEV, ${ }^{*}$ University of Haifa \\ ONNO BOXMA, ${ }^{* *}$ EURANDOM and Eindhoven University of Technology \\ GÉRARD LETAC, ${ }^{* * *}$ Université Paul Sabatier
}

\begin{abstract}
Let $f$ be a probability density function on $(a, b) \subset(0, \infty)$, and consider the class $\mathcal{C}_{f}$ of all probability density functions of the form $P f$, where $P$ is a polynomial. Assume that if $X$ has its density in $\mathcal{C}_{f}$ then the equilibrium probability density $x \mapsto \mathrm{P}(X>x) / \mathrm{E}(X)$ also belongs to $\mathcal{C}_{f}$ : this happens, for instance, when $f(x)=C \mathrm{e}^{-\lambda x}$ or $f(x)=C(b-x)^{\lambda-1}$. We show in the present paper that these two cases are the only possibilities. This surprising result is achieved with an unusual tool in renewal theory, by using ideals of polynomials.

Keywords: Renewal theory; excess lifetime; polynomial density; ideals of polynomials

2000 Mathematics Subject Classification: Primary 60K05; 13P10
\end{abstract}

\section{Introduction: equilibrium distribution}

Let $X_{1}, X_{2}, \ldots$ be a sequence of nonnegative independent random variables with a common distribution $F$, with probability density function (PDF) $f$ and Laplace-Stieltjes transform (LST) $\phi$. Letting $\mu=\mathrm{E}\left(X_{i}\right)$, it is assumed that $0<\mu<\infty$. The random variable $X_{i}$ denotes the interoccurrence time between the $(i-1)$ th and $i$ th events in some probability problem. The counting process $\{N(t), t \geq 0\}$, where $N(t)=\max \left\{n \geq 0: X_{1}+\cdots+X_{n} \leq t\right\}$, is called the renewal process generated by the interoccurrence times $X_{1}, X_{2}, \ldots$ (cf. the classical textbooks [3], [5], and [6]). An important role in renewal theory is played by the backward recurrence time $A_{t}$ (the time since the last renewal before $t$ ) and the forward recurrence time $B_{t}$ (the time until the first renewal after $t$ ). If the $X_{i}$ are interpreted as lifetimes then $A_{t}$ is the past lifetime at $t$ and $B_{t}$ is the residual or excess lifetime at $t$. It is well known that the limiting distributions of $A_{t}$ and $B_{t}$ for $t \rightarrow \infty$ are given by (with $X$ a generic random variable with distribution $F$ )

$$
\lim _{t \rightarrow \infty} \mathrm{P}\left(A_{t} \leq x\right)=\lim _{t \rightarrow \infty} \mathrm{P}\left(B_{t} \leq x\right)=\int_{y=0}^{x} \frac{\mathrm{P}(X>y)}{\mu} \mathrm{d} y .
$$

Denote this limiting or equilibrium excess lifetime distribution by $F_{\mathrm{e}}$, and its PDF by $f_{\mathrm{e}}(x)=$ $\mathrm{P}(X>x) / \mu=\int_{x}^{\infty}(f(y) / \mu) \mathrm{d} y$. Its LST is given by $\varphi_{\mathrm{e}}(s)=(1-\varphi(s)) / s \mu$. Excess lifetimes play an extremely important role in applied probability. They arise in a host of real-life problems,

\footnotetext{
Received 20 July 2009; revision received 2 November 2009.

* Postal address: Department of Statistics, University of Haifa, Haifa 31905, Israel.

Email address: barlev@stat.haifa.ac.il

** Postal address: Department of Mathematics and Computer Science, Eindhoven University of Technology, PO Box 513, 5600 MB Eindhoven, The Netherlands. Email address: boxma@win.tue.nl

*** Postal address: Laboratoire de Statistique et Probabilité, Université Paul Sabatier, 31062 Toulouse, France.

Email address: gerard.letac@alsatis.net
} 
ranging from reliability theory to inventory and queueing theory. In many queueing problems we need to know the time until completion of the ongoing service (residual service time); e.g. the residual service time plays a key role in the celebrated Pollaczek-Khintchine formula for the steady-state waiting time distribution in the M/G/1 first-come-first-served queue (see [1, Chapter VIII]). In reliability and maintenance problems, we need to know the time until breakdown of a machine, or until an ongoing repair is completed, etc. We refer the reader to Chapter 1 of [6] for a host of other examples, which confirm the importance of obtaining insight into the characteristics of the distribution of the residual lifetime.

A related important random variable is $X_{N(t)+1}$, the length of the renewal interval seen by an outside observer at $t$. Denote by $\hat{X}$ a random variable with distribution the limiting distribution of $X_{N(t)+1}$. Its steady-state PDF is $y f(y) / \mu$ and $\mathrm{P}(\hat{X}>x)=\int_{x}^{\infty}(y f(y) / \mu) \mathrm{d} y$.

In a recent report [2], it is shown that the class of distributions on the positive reals with a rational LST, also known as matrix-exponential distributions, is closed under formation of moment distributions (distributions with density $\left.y^{i} f(y) / \int_{0}^{\infty} x^{i} f(x) \mathrm{d} x\right)$. In Section 2 we also observe that, for the classes of exponential, Erlang, and hyperexponential distributions, the PDF $f_{\mathrm{e}}$, and also the PDF of $\hat{X}$, are again exponential, Erlang, hyperexponential, or mixtures of those. The beta distribution has a similar closure property. This has led us to study a much more general question: which PDFs have the property that, for any polynomial $P, \int_{x}^{b} P(t) f(t) \mathrm{d} t$ can be written in the form of a product of another polynomial and $f(x)$ ? This question is answered in our main result, Proposition 3.1 in Section 3. But first, in Section 2, we provide several examples where we demonstrate the property of Proposition 3.1. In considering these examples, it should be realized that $P(t) f(t)$ is also a PDF, up to a multiplicative constant.

\section{Examples}

In this section we consider two examples. One is related to the exponential distribution, the other to the beta distribution.

Example 2.1. (i) If $X \sim \exp (\lambda)$, i.e. $\varphi(s)=\lambda /(\lambda+s)$, then $\varphi_{\mathrm{e}}(s)=\varphi(s)$, and $f_{\mathrm{e}}(x)=f(x)$ : the residual lifetime is again exponential. Of course, this is the familiar memoryless property.

(ii) If $F$ is a hyperexponential distribution, i.e. a mixture of exponential distributions, then $F_{\mathrm{e}}$ is also hyperexponential. If $F$ is $\operatorname{Erlang}(n)$ then $F_{\mathrm{e}}$ is a mixture of $\operatorname{Erlang}(i)$ with weights $1 / n$. If $F$ is a mixture of $\operatorname{Erlang}(i)$ with weights $p_{i}, i=1, \ldots, n$, then $F_{\mathrm{e}}$ is also a mixture of $\operatorname{Erlang}(i), i=1, \ldots, n$, with different weights

$$
p_{i}^{*}=\sum_{j=i}^{n} \frac{p_{j}}{\sum_{k=1}^{n} k p_{k}}, \quad i=1, \ldots, n .
$$

In the above example $F_{\mathrm{e}}$ is either a mixture of exponential distributions or a mixture of convolutions of exponential distributions; or, equivalently, the related PDF $f_{\mathrm{e}}$ has the form

$$
f_{\mathrm{e}}(x)=\sum_{i=1}^{n} P_{i}(x) \mathrm{e}^{-\lambda_{i} x},
$$

where $n \in \mathbb{N}, P_{i}(x)$ is a polynomial in $x$, and $\lambda_{i}>0$. A similar statement holds for

$$
\mathrm{P}(\hat{X}>x)=\frac{1}{\mu} \int_{x}^{\infty} t f(t) \mathrm{d} t .
$$


It should be further noted that in Example 2.1(i) and (ii), we have a PDF of the form $P(t) f(t)$ with $P$ a polynomial and $f$ an exponential; furthermore, $\int_{x}^{b} P(t) f(t) \mathrm{d} t$ has the form of the product of another polynomial and $f$.

Example 2.2. Now consider the beta PDF

$$
f(x)=\left(\frac{x-a}{b-a}\right)^{\zeta-1}\left(\frac{b-x}{b-a}\right)^{\lambda-1} \frac{1}{(b-a) B(\zeta, \lambda)},
$$

where $B(\zeta, \lambda)=\int_{0}^{1} x^{\zeta-1}(1-x)^{\lambda-1} \mathrm{~d} x$ is the beta function. If $\zeta=1$ then

$$
f_{\mathrm{e}}(x)=\frac{(b-x)^{\lambda}}{(b-a)^{\lambda}},
$$

which is again a (special) beta PDF with $\zeta=1$. We see here a similar closure property as in the previous example. We could also have taken a weighted sum of special beta PDFs multiplied by polynomials, and it is easily seen that taking the integration $\int_{x}^{b}$ with respect to such a sum results in other polynomials multiplied by special beta PDFs.

This raises the following question. For which PDFs $f$ (or, equivalently, LSTs $\varphi$ ) is the equilibrium PDF in (1.1) a PDF in the same 'class' of PDFs as $f$, or a polynomial multiplied with $f$ ? In the next section we introduce such a closure property in a more general setting, and we prove a characterization result. If $f$ is concentrated on $0 \leq a<b \leq \infty$ then which PDFs $f$ have the property that, for any polynomial $P, \int_{x}^{b} P(t) f(t) \mathrm{d} t$ can be written in the form of a product of another polynomial and $f(x)$ ? We show in Proposition 3.1, below, that a necessary and sufficient condition for this to hold is that either $b=\infty$ and $f(x)=C \mathrm{e}^{-\lambda x}$, where $\lambda>0$ and $1 / C=\int_{a}^{b}(t-a) f(t) \mathrm{d} t$, or $b$ is finite and $f(x)=C(b-x)^{\lambda-1}$, i.e. $f$ is either exponential or of beta type.

\section{The main result}

Let $f$ be a PDF on $(a, b)$ with $0 \leq a<b \leq \infty$ such that $1 / C=\int_{a}^{b}(t-a) f(t) \mathrm{d} t<\infty$. Consider the new PDF on $(a, b)$ defined by $T(f)(x)=C \int_{x}^{b} f(t) \mathrm{d} t$. Note that, up to a multiplicative constant, this is $f_{\mathrm{e}}(x)$. For instance, if $(a, b)=(0, \infty)$, consider the class $\mathcal{F}$ of the PDFs of the form

$$
f(x)=\sum_{i=1}^{n} P_{i}(x) \mathrm{e}^{-\lambda_{i} x},
$$

where $P_{i}(x)$ is a polynomial and $\lambda_{i}>0$. Because of the formula

$$
\int_{x}^{\infty} \lambda^{n} \frac{t^{n-1}}{(n-1) !} \mathrm{e}^{-\lambda t} \mathrm{~d} t=\sum_{k=0}^{n-1} \lambda^{k} \frac{x^{k}}{k !} \mathrm{e}^{-\lambda x},
$$

clearly $T(f)$ is also in $\mathcal{F}$. A similar situation occurs when considering a bounded interval $(a, b)$ and the class $g$ of PDFs on $(a, b)$ which are polynomials $P$ multiplied by the function $f(x)=(b-x)^{\lambda-1}$, where $\lambda>0$. Here, $g$ is stable by $T$, meaning that $T(g) \subset g$ (write $P(x) f(x)$ in the form $\sum_{k=0}^{n} p_{k}(b-x)^{k+\lambda-1}$ to be convinced of this fact). Of course, choosing a class $\mathcal{C}$ of PDFs on $(a, b)$ having all their moments implies that the class of PDFs defined by

$$
\mathcal{C}_{1}=\bigcup_{n=0}^{\infty} T^{n}(\mathcal{C})
$$


is stable by $T$. But we are going to show that the classes $\mathcal{F}$ and $\mathcal{G}$ above are unique in the following sense.

Proposition 3.1. Let $f$ be a positive measurable function on $(a, b)$ with $0 \leq a<b \leq \infty$ such that $\int_{a}^{b} t^{n} f(t) \mathrm{d} t<\infty$ for any nonnegative integer $n$. Suppose that, for any polynomial $P$, there exists a polynomial $A(P)$ such that, for all $x \in(a, b)$, we have

$$
\int_{x}^{b} P(t) f(t) \mathrm{d} t=A(P)(x) f(x) .
$$

Then there exist $C, \lambda>0$ such that either $b$ is infinite and $f(x)=C \mathrm{e}^{-\lambda x}$, or $b$ is finite and $f(x)=C(b-x)^{\lambda-1}$.

Remarks. The statement of Proposition 3.1 describes the few functions $f$ on $(a, b)$ such that the class $\mathcal{C}_{f}$ of PDFs of the form $P(x) f(x)$ is stable by the operation $T$ described above, with $T(P f)=A(P) f$. Note that in both cases $a$ is not necessarily 0 . For instance, if $f(x)=\mathrm{e}^{-\lambda x}$ on $(a, b)=(a, \infty)$ and $P(x)=\lambda^{n} x^{n-1} /(n-1)$ !, we have (cf. (3.1))

$$
A(P)(x)=\sum_{k=0}^{n-1} \frac{\lambda^{k} x^{k}}{k !} .
$$

Note that $A(1)=1 / \lambda$. Since $A$ is a linear operator, these formulae describe $A$ completely. Similarly, if $f$ is $(b-x)^{\lambda-1}$ on the bounded interval $(a, b)$ and if $P(x)=(b-x)^{n}$, we have

$$
A(P)(x)=\frac{(b-x)^{n+1}}{n+\lambda} .
$$

For instance, $A(1)=(b-x) / \lambda$.

Let us also insist on the fact that the proposition describes the only two possibilities. We could be tempted if $f$ satisfies (3.2) to coin the new function $f_{1}(x)=R(x) f(x)$, where $R$ is a nonconstant polynomial which is positive on $(a, b)$, and to observe that, for all polynomials $P$, we have

$$
\int_{x}^{b} P(t) f_{1}(t) \mathrm{d} t=\frac{A(P R)(x)}{R(x)} f_{1}(x) .
$$

A consequence of the proposition is that it is impossible that $R$ divides $A(P R)$ for all polynomials $P$.

\subsection{Proof of Proposition 3.1}

For $P \equiv 1$, we define $Q(x)=A(1)(x)$. Writing $\int_{x}^{b} f(t) \mathrm{d} t=Q(x) f(x)$ shows that the polynomial $Q$ must be positive on $(a, b)$. Since $f$ is integrable, writing

$$
f(x)=\frac{1}{Q(x)} \int_{x}^{b} f(t) \mathrm{d} t
$$

shows that $f$ must be continuous and differentiable, and, thus, infinitely differentiable. Now taking the derivative in $x$ of $\int_{x}^{b} P(t) f(t) \mathrm{d} t=A(P)(x) f(x)$ gives the differential equation

$$
-P(x) f(x)=A(P)^{\prime}(x) f(x)+A(P)(x) f^{\prime}(x),
$$


which we rewrite as

$$
\frac{f^{\prime}(x)}{f(x)}=-\frac{P(x)+A(P)^{\prime}(x)}{A(P)(x)} .
$$

Note that, since the left-hand side of this equation does not depend on $P$, we can get information on $A(P)$ by replacing $P$ with 1 , giving the following differential equation in $A(P)$ :

$$
\frac{P(x)+A(P)^{\prime}(x)}{A(P)(x)}=\frac{1+Q^{\prime}(x)}{Q(x)} .
$$

As a consequence, all information on $f$ and $A(P)$ is actually given by the polynomial $Q$.

Case 1: $Q$ of degree 0 . If $Q$ is the nonzero constant $1 / \lambda$, the equation $f^{\prime} / f=-\left(1+Q^{\prime}\right) / Q$ gives $f(x)=\mathrm{e}^{-\lambda x}$ on $(a, b)$. If $b=\infty$, we have already seen that, if $\lambda>0$, the identity $\int_{x}^{b} P(t) f(t) \mathrm{d} t=A(P)(x) f(x)$ holds for a suitable operator $A$ defined by (3.3). If $\lambda \leq 0$, the condition $\int_{a}^{b} t^{n} f(t) \mathrm{d} t<\infty$ is not fulfilled. If $b<\infty$ then $\int_{x}^{b} P(t) f(t) \mathrm{d} t=A(P)(x) f(x)$ does not hold since, for $P=\lambda$, we obtain

$$
\int_{x}^{b} \lambda \mathrm{e}^{-\lambda t} \mathrm{~d} t=\mathrm{e}^{-\lambda x}-\mathrm{e}^{-\lambda b}
$$

which is not of the desired form of a polynomial multiplied by $\mathrm{e}^{-\lambda x}$.

Case 2: $Q$ of degree 1. If $Q$ is a first-degree polynomial, we write it as $Q(x)=\left(b_{1}-x\right) / \lambda$, where $b_{1}$ is a real number and $\lambda$ is a nonzero number. From the equation $f^{\prime} / f=-\left(1+Q^{\prime}\right) / Q$ on $(a, b)$ and the fact that $(\mathrm{d} / \mathrm{d} t) \log |t|=1 / t$, we find that $f(x)=C\left|b_{1}-x\right|^{\lambda-1}$ for some positive number $C$. Suppose that $b=\infty$. Clearly, $\int_{a}^{\infty} t^{n} f(t) \mathrm{d} t<\infty$ is impossible if $n$ is large enough. Thus, $b<\infty$. Now, for all $x$ in $(a, b)$, we have

$$
\int_{x}^{b}\left|b_{1}-t\right|^{\lambda-1} \mathrm{~d} t=\left|b_{1}-x\right|^{\lambda-1} \frac{b_{1}-x}{\lambda}=\frac{\left|b_{1}-x\right|^{\lambda}}{|\lambda|} .
$$

Since the left-hand side must converge to 0 when $x \rightarrow b$, this would imply that $b=b_{1}$ and that $\lambda>0$.

Case 3: $Q$ of degree greater than or equal to 2 . We now claim that $Q$ has necessarily degree less than or equal to 1, a more difficult part of the proof. Suppose that $Q$ has degree greater than or equal to 2, and suppose that the differential equation $Q P=\left(1+Q^{\prime}\right) Y-Q Y^{\prime}$ always has a polynomial solution $Y=A(P)$ for any polynomial $P$.

To reach a contradiction, we introduce the following notation. We denote by $\mathcal{A}$ the algebra of polynomials with real coefficients. If $A \in \mathcal{A}$, we denote by $\ell_{A}$ the ideal generated by $A$, that is, the set of polynomials divisible by $A$ :

$$
\ell_{A}=\{A P ; P \in \mathcal{A}\} .
$$

Recall that in general an ideal of $\mathcal{A}$ is a linear subspace $\ell$ of $\mathcal{A}$ such that $P B$ is in $\ell$ for any $B \in \ell$ and any $P \in \mathcal{A}$. The important result is that in this algebra $\mathcal{A}$ of polynomials, for any ideal $\ell$, there exists an $A \in \mathcal{A}$ such that $\ell=\ell_{A}$ : this is called the principal ideal property; see, e.g. [4, p. 105].

Finally, we introduce the notation for the linear application $\varphi$ of $\mathcal{A}$ into itself, defined by

$$
Y \mapsto \varphi(Y)=\left(1+Q^{\prime}\right) Y-Q Y^{\prime}
$$

Assuming that $Q P=\varphi(Y)$ has a solution $Y$ in $\mathcal{A}$ for each $P \in \mathcal{A}$ is equivalent to saying that the image $\varphi(\mathcal{A})$ of $\varphi$ contains the ideal $\ell_{Q}$. 
Lemma 3.1. Let $B_{0}$ and $C_{0}$ be two polynomials, and consider the linear application $\varphi_{0}$ of $\mathcal{A}$ into itself, defined by

$$
\varphi_{0}(Y)=B_{0} Y-C_{0} Y^{\prime}
$$

We assume that $\varphi_{0}(\mathcal{A}) \supset \ell_{C_{0}}$. Then there exist $A_{1}, B_{1}, C_{1} \in \mathcal{A}$ such that $\varphi_{0}(\mathcal{A})=\ell_{A_{1}}$, $B_{0}=A_{1} B_{1}$, and $C_{0}=A_{1} C_{1}$. Furthermore, if $\varphi_{1}(Y)=B_{1} Y-C_{1} Y^{\prime}$, we have $\varphi_{1}(\mathcal{A}) \supset \ell_{C_{1}}$.

Proof. We show that $\varphi_{0}(\mathcal{A})$ is an ideal. Since $\varphi_{0}$ is linear, the set $\varphi_{0}(\mathcal{A})$ is a linear subspace of $\mathcal{A}$. Thus, we want to show that if $B=\varphi\left(Y_{0}\right)$ and $P$ are arbitrary elements of $\varphi_{0}(\mathcal{A})$ and $\mathcal{A}$, respectively, then the polynomial $P \varphi_{0}\left(Y_{0}\right)$ is in $\varphi_{0}(\mathcal{A})$. Since $\varphi_{0}(\mathcal{A}) \supset \ell_{C_{0}}$, there exists $Y_{1} \in \mathcal{A}$ such that $\varphi_{0}\left(Y_{1}\right)=C_{0} P^{\prime} Y_{0}$. Thus,

$$
\begin{aligned}
\varphi_{0}\left(P Y_{0}+Y_{1}\right) & =\varphi_{0}\left(P Y_{0}\right)+\varphi_{0}\left(Y_{1}\right) \\
& =B_{0} P Y_{0}-C_{0} P Y_{0}^{\prime}-C_{0} P^{\prime} Y_{0}+\varphi_{0}\left(Y_{1}\right) \\
& =B_{0} P Y_{0}-C_{0} P Y_{0}^{\prime} \\
& =P \varphi_{0}\left(Y_{0}\right) .
\end{aligned}
$$

Equality $P \varphi_{0}\left(Y_{0}\right)=\varphi_{0}\left(P Y_{0}+Y_{1}\right)$ shows that $\varphi_{0}(\mathcal{A})$ is an ideal of $\mathcal{A}$. From the principal ideal property, there exists $A_{1}$ such that $\varphi_{0}(\mathcal{A})=\ell_{A_{1}}$. Since $\ell_{A_{1}} \supset \ell_{C_{0}}, A_{1}$ divides $C_{0}$. Thus, there exists $C_{1}$ such that $C_{0}=A_{1} C_{1}$. Since $\varphi_{0}(Y)=B_{0} Y-C_{0} Y^{\prime}=B_{0} Y-A_{1} C_{1} Y^{\prime}$ is a multiple of $A_{1}$ for any $Y$, then $B_{0}=\varphi_{0}(1)=A_{1} B_{1}$ is also a multiple of $A_{1}$. Finally, since, for each $P$, there exists $Y$ such that

$$
\varphi_{0}(Y)=B_{0} Y-C_{0} Y^{\prime}=C_{0} P=A_{1} B_{1} Y-A_{1} C_{1} Y^{\prime}=A_{1} C_{1} P,
$$

this implies that the same $Y$ satisfies $\varphi_{1}(Y)=B_{1} Y-C_{1} Y^{\prime}=C_{1} P$, showing that $\varphi_{1}(\mathcal{A}) \supset \ell_{C_{1}}$. This completes the proof of Lemma 3.1.

We now iterate Lemma 3.1. For each $n=1,2, \ldots$, there exist $A_{n}, B_{n}$, and $C_{n}$ such that

$$
B_{0}=A_{1} A_{2} \cdots A_{n} B_{n}, \quad C_{0}=A_{1} A_{2} \cdots A_{n} C_{n},
$$

and such that if we define $\varphi_{n}(Y)=B_{n} Y-C_{n} Y^{\prime}$, we have $\varphi_{n}(\mathcal{A})=\ell_{A_{n+1}} \supset \ell_{C_{n}}$. In particular, $\sum_{k=1}^{n} \operatorname{deg} A_{k} \leq \operatorname{deg} C_{0}$ implies that, for large enough $n, \operatorname{deg} A_{n+1}=0$. As a consequence, $A_{n+1}$ must be a constant polynomial, which is equivalent to saying that $\varphi_{n}$ is surjective.

We now apply the above considerations to the particular case where $B_{0}=1+Q^{\prime}$ and $C_{0}=Q$, where $Q$ is a polynomial of degree $d_{0} \geq 2$. Thus, $\varphi=\varphi_{0}$ in the lemma. With this choice of $\left(B_{0}, C_{0}\right)$, we show that whatever $n$ is, the map $\varphi_{n}$ cannot be surjective when the degree of $Q$ is greater than or equal to 2 . Write

$$
Q(x)=C_{0}(x)=c_{0} x^{d_{0}}+\text { lower-degree terms },
$$

and, more generally,

$$
C_{n}(x)=c_{n} x^{d_{n}}+\text { lower-degree terms, } \quad B_{n}(x)=b_{n} x^{d_{n}-1}+\text { lower-degree terms. }
$$

We show by induction on $n$ that $b_{n}=d_{0} c_{n}$. This is obvious for $n=0$ since $B_{0}=1+Q^{\prime}$ and $d_{0} \geq 2$. Suppose that it is true for $n-1$. Since $B_{n-1}=A_{n} B_{n}$ and $C_{n-1}=A_{n} C_{n}$, and if the term of maximum degree of $A_{n}$ is $a_{n} x^{m}$, then $d_{n-1}=d_{n}+m, b_{n-1}=a_{n} b_{n}$, and $c_{n-1}=a_{n} c_{n}$. Since, by definition, $a_{n} \neq 0$, the equality $b_{n}=d_{0} c_{n}$ holds. 
We finally use this fact to prove that $\varphi_{n}$ cannot be surjective, by showing that there is no $Y$ such that

$$
\varphi_{n}(Y)(x)=B_{n}(x) Y(x)-C_{n}(x) Y^{\prime}(x)=x^{d_{0}+d_{n}-1}
$$

holds. Suppose that there exists such a $Y$, with highest degree term $\alpha x^{m}$. The highest degree term of $B_{n} Y-C_{n} Y^{\prime}$ is $\left(d_{0}-m\right) \alpha c_{n} x^{d_{n}+m-1}$ if $m \neq d_{0}$, which cannot be equal to $x^{d_{0}+d_{n}-1}$. If $m=d_{0}$, the highest degree term of $B_{n} Y-C_{n} Y^{\prime}$ has degree less than $d_{0}+d_{n}-1$. We obtain the desired contradiction. This completes the proof of Proposition 3.1.

Remark. As observed by the referee about the statement of Proposition 3.1, positivity of $f$ can be replaced by a slightly weaker hypothesis: (i) nonnegativity of $f$; (ii) if $G\left(x_{0}\right)$ is the Lebesgue measure of the set $\left\{x_{0} \leq x<b ; f(x)>0\right\}$ then $G\left(x_{0}\right)>0$ for all $x_{0} \in(a, b)$. The equality $\int_{x}^{b} f(t) \mathrm{d} t=Q(x) f(x)$ shows that this weaker hypothesis implies the positivity of $f$.

\section{References}

[1] Asmussen, S. (2003). Applied Probability and Queues, 2nd. edn. Springer, New York.

[2] Bladt, M. and Nielsen, B. F. (2009). Moment distributions of phase type. Submitted.

[3] Cox, D. R. (1962). Renewal Theory. Methuen, London.

[4] Herstein, I. N. (1964). Topics in Algebra. Blaisdell Publishing, New York.

[5] Ross, S. M. (1996). Stochastic Processes, 2nd edn. John Wiley, New York.

[6] TiJms, H. C. (1994). Stochastic Models. An Algorithmic Approach. John Wiley, Chichester. 